\title{
The Crosstalk Between Cancer Cells and
}

\section{Neutrophils Enhances Hepatocellular Carcinoma Metastasis via Neutrophil Extracellular Traps-Associated Cathepsin G Component: A Potential Therapeutic Target}

\section{Xiangqian Guan \\ Yuyan Lu \\ Heping Zhu \\ Shuqi Yu \\ Wenxiu Zhao \\ Xiaoqin Chi \\ Chengrong Xie \\ Zhenyu Yin}

Department of Hepatobiliary Surgery, Zhongshan Hospital, Xiamen University, Fujian Provincial Key Laboratory of Chronic Liver Disease and Hepatocellular Carcinoma, Xiamen, Fujian, People's Republic of China
Correspondence: Chengrong Xie; Zhenyu Yin

Department of Hepatobiliary Surgery, Zhongshan Hospital, Xiamen University, Fujian Provincial Key Laboratory of Chronic Liver Disease and Hepatocellular Carcinoma, 209 South Hubin Road, Xiamen, Fujian Province, 361004, People's Republic of China

Email eason_xiecr@I26.com;

yinzy@xmu.edu.cn
Background: Emerging evidences have highlighted the roles of neutrophils, as the major host microenvironment component, in the development of hepatocellular carcinoma (HCC). Neutrophils extracellular traps (NETs) produced in the infection can strengthen the behavior of cancer metastasis. Here, we investigated the roles of NETs in HCC metastasis and further explore the underlying mechanism of how NETs interact with cancer.

Methods: The neutrophils were isolated from whole blood of HCC patients and used to evaluate the formation of NETs. NET markers were detected in tissue samples, plasma and cell climbing slice. Mouse models were used to evaluate the roles of NETs in HCC metastasis in vivo, and the corresponding mechanisms were explored using in vivo and in vitro assays.

Results: An increase in the release of NETs in patients with HCC, particularly those with portal vein tumor thrombosis (PVTT). The presence of NETs in HCC tumor tissues closely correlated with a poor prognosis. Functionally, the invasion ability of HCC cells was enhanced by coculture with HCC neutrophils, through NETs formation, while the neutrophils from a healthy donor (HD) exhibited the inhibition of the invasion ability. Furthermore, we observed an enhanced ability of forming NETs in neutrophils from HCC patients in vitro, especially patients with PVTT or extra-hepatic metastasis. An in-vivo animal study demonstrated that neutrophils of HCC facilitated the metastatic behavior towards the lung. The further mechanistic investigation unveiled that HCC cells-derived cytokine IL-8 triggered NETs formation in an NADPH oxidasedependent manner, and NETs-associated cathepsin $\mathrm{G}(\mathrm{cG})$ promoted HCC metastasis in vitro as well as vivo. Clinically, the expression of the $\mathrm{cG}$ protein in tumor tissues displayed a close correlation with the disease prognosis of HCC patients.

Conclusion: Our findings implicated that the induction of NETs by HCC cells is a critical metastasis-supporting cancer-host interaction and that NETs may serve as an immune-based potential therapeutic target against HCC progression.

Keywords: hepatocellular carcinoma, neutrophils extracellular traps, cathepsin G, IL-8, E-cadherin, metastasis, NADPH

\section{Introduction}

Despite the significant progress has been made in its diagnosis and treatment in recent years, hepatocellular carcinoma (HCC) remains one of the leading causes of cancer-related death worldwide. ${ }^{4,6,7}$ Systemic therapies for HCC, such as surgical 
resection, transarterial chemoembolization, local ablation, and targeted molecular therapy, have markedly advanced; however, the survival rate remains low, mainly due to distant metastasis. ${ }^{12,35}$ Thus, a better understanding of the molecular mechanism underlying HCC metastasis is urgently required.

The inflammatory tumor microenvironment is crucial in the development of HCC. ${ }^{11,12}$ Single-cell sequencing analyses have revealed a landscape of infiltrating $\mathrm{T}$ cells and their functional properties in HCC tissues. ${ }^{45}$ Myeloid derived suppressor cells (MDSCs) have been demonstrated to accumulate in several animal models of $\mathrm{HCC}^{27,28}$ Tumor-infiltrating leukocytes represent a significant proportion in the inflammation microenvironment, and influence nearly all steps in the progression of malignancies, including metastasis. ${ }^{13,14,17}$ As a critical element among leukocytes, neutrophils serve as a paradigm for the interaction between inflammation and cancer. ${ }^{7,9,11,24}$ The infiltration of tumor-associated neutrophils (TAN) and the elevated neutrophil-lymphocyte ratio are correlated with poor outcomes in HCC. ${ }^{12,13}$ Neutrophils exhibit several functions, including phagocytosis, generation of reactive oxygen species, as well as the formation of neutrophils extracellular traps (NETs), which are DNA meshes with associated cytotoxic enzymes released into the extracellular space. ${ }^{8,18}$ NETs represent a novel aspect of neutrophil biology and were initially discovered as a part of the process of innate immune defense to kill microorganisms. ${ }^{46,47}$

In the past decades, NETs formation has been closely associated with several cancer-related diseases. ${ }^{22,23}$ NETs bridge the crosstalk between cancer cells and neutrophils. $^{3,5,48}$ Recent pieces of evidence suggest cancer cells-secreted factors trigger NETs formation, which in turn promotes cancer metastasis by inducing a pro-inflammatory response in tumor cells. ${ }^{12,40,47}$ Emerging evidence shows that NETs-associated proteases, such as neutrophil elastase (NE) and matrix metalloproteinase 9, support metastasis colonization in the lung by remodeling the extracellular matrix. ${ }^{5}$ Neutrophils also degranulate to release Ser proteases, such as cathepsin G (cG), to degrade thrombospondin-1, thereby enhancing metastatic outgrowth. ${ }^{20}$ To date, the effect of NETs, especially the associated proteases, on HCC progression, remains poorly understood.

In the present study, we examined the expression level of NETs-CitH3 in HCC patient samples and assessed the relationship between CitH3 expression and the clinicopathological features with HCC. Furthermore, we explored the ability of cancer cells to induce NETs formation, in turn, the effect of NETs on HCC metastasis behavior, and also how the NETs-associated cG contributed to this process. To summarize, our study revealed a previously unknown mechanism underlying the crosstalk between HCC and neutrophils, which would exhibit critical clinical implications.

\section{Patients and Methods}

\section{Neutrophils Isolation}

For human neutrophils isolation, EDTA-K $\mathrm{K}_{2}$ anticoagulation vein whole blood samples were obtained preoperatively from patients with $\mathrm{HCC}$ at Zhongshan Hospital. Approximately $10 \mathrm{~mL}$ of fasting peripheral blood was withdrawn from the selected patients in the morning. The blood sample was placed in the $0.25 \%$ ethylenediaminetetraacetic acid (EDTA) anticoagulant inside a tube for neutrophil separation. A double gradient was formed by layering an equal volume of Histopaque (Sigma)-1.077 over 1.119. The whole blood was carefully layered onto an equal volume of double Histopaque gradient (1.077 and 1.119). After centrifugation at room temperature, erythrocytes were aggregated and rapidly sediment. The neutrophils fraction was collected from the 1.077-1.119 interface. The neutrophils were isolated and extracted from the whole blood in less than $2 \mathrm{~h}$.

\section{Tissue Collection}

Eighty-five HCC tissues were obtained from patients with HCC who underwent hepatectomy between 2011 and 2019 at Zhongshan Hospital of Xiamen University. Tissue samples were immediately frozen in liquid $\mathrm{N}_{2}$ and stored at $-80^{\circ} \mathrm{C}$ for further experiments. Written informed consent for participation in this research was obtained from all patients. The consent and experimental procedures were approved by the ethics committee of Zhangshan Hospital of Xiamen University according to the World Medical Association Declaration of Helsinki.

\section{Serum NETs Detection}

Human serum NETs marker myeloperoxidasedeoxyribonucleic acid (MPO-DNA) was measured using a capture ELISA assay with some modification. In brief, 5 $\mu \mathrm{g} / \mathrm{mL}$ MPO monoclonal antibody (1:100, ab4704, Abcam) was coated into a 96-well plate, followed by overnight incubation at $4^{\circ} \mathrm{C}$ overnight. After blocking with $1 \%$ bovine serum albumin (BSA), serum was added into each well, followed by incubation at room temperature for $3 \mathrm{~h}$. After washing four times with phosphate- 
buffered saline (PBS), PicoGreen ${ }^{\circledR}$ dsDNA Quantitation Reagent was added into each sample according to the manufacturer's instructions, followed by incubation at room temperature for $5 \mathrm{~min}$, protected from light. The values were then measured using instrument parameters with a filter setting of $480 \mathrm{~nm} / 520 \mathrm{~nm}$ excitation/emission.

\section{Immunohistochemistry Staining}

Briefly, tissues were fixed with $10 \%$ neutral formalin, embedded in paraffin, and then $4 \mu \mathrm{m}$-thick sections were prepared. The sections were deparaffinized, hydrated, and soaked in $3 \% \mathrm{H}_{2} \mathrm{O}_{2}$ for $1 \mathrm{~h}$, and then blocked with $5 \%$ BSA at room temperature overnight. The sections were incubated with anti-histone $\mathrm{H} 3$ (citrulline $\mathrm{R}_{2}+\mathrm{R}_{8}+\mathrm{R}_{17}$, CitH3) (1:500, ab5103, Abcam), anti-Ly6G (1:100, ab25377, Abcam), anti-cathepsin G (1:200, ab192793, Abcam) or anti-IL-8 (1:150, \#44133, Signalway Antibody) antibodies at $4{ }^{\circ} \mathrm{C}$ overnight. The slides were incubated with biotinylated antibodies for $1 \mathrm{~h}$ and stained with diaminobenzidine (DAB; Maixin Biotechnology, Fuzhou, China), followed by counterstaining with hematoxylin (Maixin Biotechnology).

\section{Immunofluorescence}

Immunofluorescence assay was performed to determine NETs formation in mice lung frozen sections, or neutrophils on a coverslip. NETs specific marker was stained with a primary antibody against anti-histone $\mathrm{H} 3$ (citrulline $\mathrm{R}_{2}+\mathrm{R}_{8}+\mathrm{R}_{17}$, CitH3) (1:400, ab5103, Abcam). After washing with PBS, the sections were stained with fluorochrome-conjugated secondary antibodies (1:200, AS007, ABclonal) and finally stained with DAPI (Invitrogen, $0.05 \mathrm{mg} / \mathrm{mL}$ ). All negative controls were designed without the primary antibody. The extension of NETs release was assessed by calculating the area occupied by $\mathrm{CitH} 3$ color spot under each confocal microscope visual field. For NETs area quantification, Image $J$ software and the particle analyzer plugin were used. Particle analyzer plugin was used to form the images of $\mathrm{CitH} 3$ color spot, and Image $\mathbf{J}$ software could calculate the percent of area occupied by $\mathrm{CitH} 3$ color.

\section{Cell Culture}

HCC cell lines (HuH7, MHCC-97L and Hepa1-6) were amplified in our laboratory and stored in liquid nitrogen to ensure that the cells used for experiments were passaged for fewer than six months. The authentication of these cell lines was performed through comparisons with the STR database. The cells were maintained in high glucose Dulbecco's modified eagle medium (DMEM) supplemented with $10 \%$ fetal bovine serum and $1 \%$ penicillin/streptomycin at $37^{\circ} \mathrm{C}$ with $5 \% \mathrm{CO}_{2}$ in the air.

\section{Transwell Assay}

For the transwell assay, approximately $5 \times 10^{5}$ speciesmatched HD or HCC neutrophils were mixed in $500 \mu \mathrm{L}$ serum-free DMEM medium, seeded onto the bottom chamber, supplemented with $10 \%$ fetal bovine serum (FBS), and then incubated for 30 min before the addition of DNaseI (Invitrogen, AM2222), NE inhibitor (MedChemExpress, 150374-95-1) or cG inhibitor (SigmaAldrich, 219372). The indicated $4 \times 10^{5} \mathrm{HCC}$ cells in $200 \mu \mathrm{L}$ serum-free DMEM medium were seeded onto an $8 \mu \mathrm{m}$ pore polycarbonate membrane with Matrigel in the upper chamber as per the manufacturer's instructions (Corning Incorporated; New York, NY, USA). To digest the structure of the NETs, $15 \mu \mathrm{L}$ DNaseI alone was added into the lower chamber culture medium. For PAD4 inhibition with GSK484, isolated neutrophils were pre-treated with the PAD4 inhibitor GSK484 (Cayman Chemical, 17488) at $37^{\circ} \mathrm{C}$ for $4 \mathrm{~h}$ before seeding onto the bottom chamber. NETs-CM was added to the bottom chamber, supplemented with $10 \%$ FBS, and $4 \times 10^{5}$ HCC cells were seeded in a serum-free DMEM medium to the upper chamber. DNaseI or cG inhibitor was added into the conditioned medium (CM) following the previous instruction.

After $24 \mathrm{~h}$ of incubation, the non-invasive cells from the upper surface of the membrane were wiped off, while the cells on the bottom surface of the membrane were fixed with 4\% paraformaldehyde (PFA) and stained with hematoxylin. The cells in ten randomly selected views were counted.

\section{Immunoblot Analysis}

Cells were lysed with the radioimmunoprecipitation assay (RIPA) lysis buffer (Beyotime; Beijing, China). Protein concentration in the lysates was measured with a BCA Protein Assay Kit (ThermoFisher). Protein samples were separated by sodium dodecyl sulfate-polyacrylamide gel electrophoresis (SDS-PAGE) and then transferred onto polyvinylidene difluoride (PVDF) membranes (Millipore). Membranes were blocked with 5\% non-fat milk (Bio-Rad) at room temperature for $1 \mathrm{~h}$, and then incubated with anti-E-cadherin (1:400, \#3195, Cell Signaling) or anti-IL-8 (1:100, ab18672, Abcam) at $4^{\circ} \mathrm{C}$ overnight, followed by incubation with anti-rabbit or anti- 
mouse secondary antibodies conjugated to horseradish peroxidase (HRP, Jackson). Immunoreactive proteins were subsequently visualized using the enhanced chemiluminescence detection system (Millipore).

\section{Preparation of NETs}

Neutrophils were isolated and seeded onto 24-well plates $\left(2.5 \times 10^{5}\right.$ cells/well). Phorbol 12-myristate 13-acetate (PMA, $500 \mathrm{nM}$, Sigma-Aldrich) was added to the isolated neutrophils, followed by incubated at $37^{\circ} \mathrm{C}$ and $5 \% \mathrm{CO}_{2}$ for 4 hours to induce NETs generation. Then, the supernatants were obtained carefully and washed twice to eliminate residual PMA or NETs-associated substances without disturbing the NETs. The supernatants containing NETs were collected and centrifuged to eliminate cell debris. Isolated NETs were stored at $-80^{\circ} \mathrm{C}$ for subsequent experiments.

\section{NETs Formation Study}

In HCC cells-CM and patients serum-induced NETs formation assays, neutrophils were incubated with the corresponding CM (1:2) for $24 \mathrm{~h}$, or incubated with serum from HCC patients or HD for $24 \mathrm{~h}$. The NOX2 inhibitor apocynin was added into the culture medium at a final concentration of $10 \mu \mathrm{M}$. IL-8 neutralizing antibody was added into culture medium at the concentration of $5 \mu \mathrm{g} / \mathrm{mL}$. For quantification, NETs on coverslips in 24-well plates were detected by immunofluorescence following the previously described instructions. The extension of NETs release was assessed by calculating the area percent of $\mathrm{CitH} 3$ color with Image $\mathbf{J}$ software.

\section{Animal Study}

Male BALB/c nude mice (6-8 weeks old, weight 20-25 g) and male C57BL/6J mice (5-6 weeks old, weight 19-23 g) were used in animal studies and fed in the experimental animal center for specific pathogen-free animals at Xiamen University. For establishing the animal model, $5 \times 10^{6} \mathrm{HuH} 7$ or $97 \mathrm{~L}$ cells were injected intravenously into nude mice. A co-injection experiment was performed by injecting $4 \times 10^{6} \mathrm{HuH} 7$ cells with $4 \times 10^{5}$ human neutrophils into nude mice via the tail vein. For male C57BL/6J mice, treatment of DNaseI ( $50 \mu \mathrm{L} /$ mouse) or cG inhibitor was given daily via intraperitoneal injection. The dose of the inhibitor was $12 \mathrm{mg} / \mathrm{kg}$ (Sigma-Aldrich, 219372). Hepa1-6 cells $\left(2 \times 10^{6}\right)$ were intravascularly injected to establish the metastatic model at two days after beginning treatment administration. Metastatic burden was assessed by calculating the area percentage of lung tissue covered by the tumor. In the H\&E-stained histological sections, the tumor-occupied area was quantitatively assessed by Image $\mathrm{J}$ software. All animal experiments were approved by the Animal Care and Use Committee of Xiamen University.

\section{Statistical Analysis}

All statistical analyses were performed using GraphPad Prism 6 software. Study data were analyzed using twosided t-tests or unpaired $t$-test. Pearson's correlation test was used for correlation analysis and log-rank (MantelCox) test was used for follow-up data and prognosis analysis. A P-value less than 0.05 indicated statistical significance.

\section{Determination of the Dose of Treatments in Animal Studies}

The dose of DNaseI in vivo was determined according to previously reported studies in the aspect of NETsosis (50 $\mu \mathrm{L} /$ mouse, via intraperitoneal injection). The dose of $\mathrm{cG}$ inhibitor in vivo (Sigma-Aldrich, 219372) was determined according to the inhibitor ((MCE, Sivelestat sodium tetrahydrate) of another NETs-carrying neutrophil elastase (NE) protease in vitro and vivo. The dose of NE inhibitor was reported to be $0.00456 \mathrm{~g} / \mathrm{L}$ in vitro, and $0.05 \mathrm{mg} / \mathrm{g}$ in mouse. Accordingly, when $\mathrm{cG}$ inhibitor was reported at $0.00124 \mathrm{~g} / \mathrm{L}$ in vitro, a dose of $\mathrm{cG}$ inhibitor at about $0.012 \mathrm{mg} / \mathrm{g}$ was used in mouse.

\section{Results}

An Increase in NETs Formation Indicates a Poor Prognosis of HCC Patients Associated with PVTT

When forming NETs, neutrophils release chromatin DNA associated with other neutrophil-derived proteins into extracellular space. $^{48}$ We examined the serum MPODNA level, a NETs product, of 30 preoperative patients with HCC and 30 healthy controls (HD) (Table S3). Our results suggested that serum MPO-DNA level increased in patients with HCC compared to HD. Among $90 \mathrm{HCC}$ patients, further investigation indicated a remarkably higher level of MPO-DNA in HCC patients with portal vein tumor thrombosis (PVTT) or extra-hepatic metastasis compared to those without metastasis (Figure 1A). Indeed, during NETs formation, histone citrullination induced by peptidyl arginine deiminases (PADs) is an important posttranslational modification that facilitates chromatin 
A

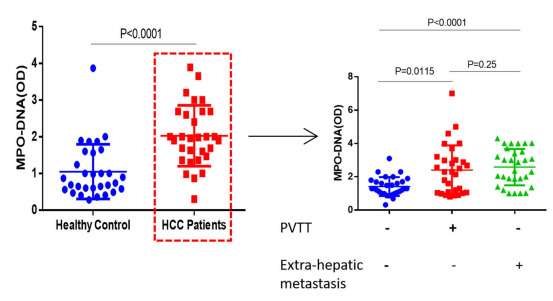

log-rank(Mantel-Cox) test

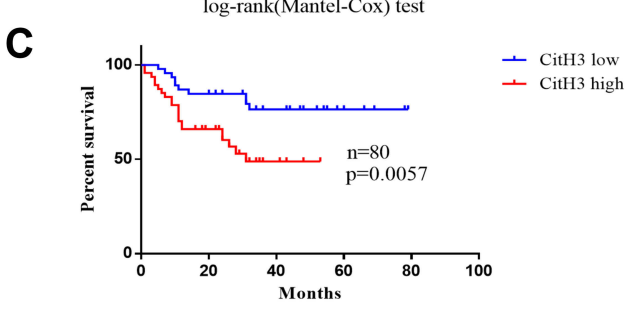

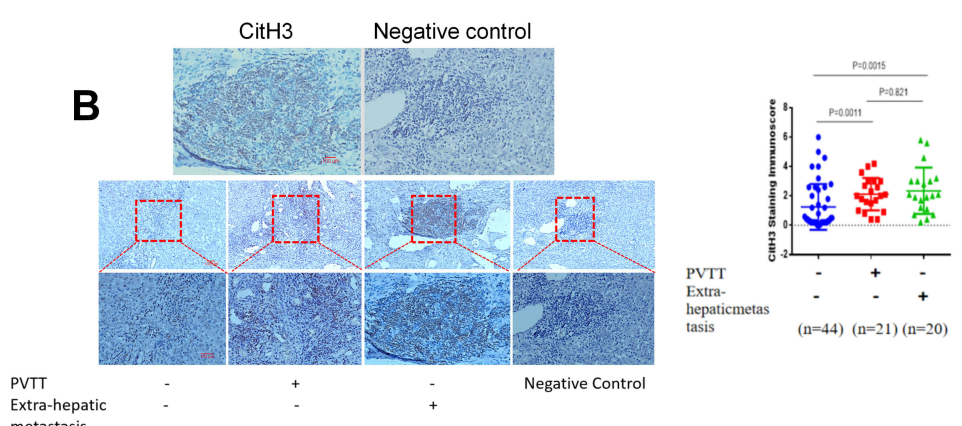

Extra-hepatic
metastasis

Figure I Increased NETs marker in HCC patients. (A) The Levels of MPO-DNA in HCC or healthy control serum samples were examined by the capture ELISA assay $(n=90)$. The red dotted box points to HCC patients, indicating that MPO-DNA levels were particularly elevated in either PVTT or extra-hepatic metastasis among HCC patients. (B) The deposition of NETs-CitH3 in 85 HCC tissue samples were examined by the immunohistochemistry assay. NETs-CitH3 level elevated in HCC patients tissue with portal vein tumor thrombosis/extra-hepatic metastasis $(n=4 I)$, compared to those without metastasis $(n=44)$. Representative images of CitH3 in human tissues were shown. Scare bar: 100um. (C) Presence of NETs-CitH3 in HCC tissues correlated with disease prognosis. Log-rank (Mantel-Cox) disease-free survival curves of HCC patients was used basing on tissue $\mathrm{CitH} 3$ immunoscore.

decondensation. Citrullinated histone $\mathrm{H} 3$ (CitH3) has been shown to be highly produced during NETsosis process mainly by peptidyl arginine deiminases 4 (PAD4), an enzyme which is crucial to NETs formation. CitH3 has been proposed as a target biomarker reflecting NETs formation, and several methods to quantify NETs in plasma samples and tissues through marker $\mathrm{CitH} 3$ have been developed. ${ }^{50,51}$ By assessing 85 patients who underwent liver resection between 2011 and 2019, we gained histological evidence for CitH3 deposition in HCC tissues, suggesting that significantly stronger $\mathrm{CitH} 3$ expression was observed in the tissues specimens from HCC patients with PVTT or extra-hepatic metastasis than in those without metastasis (Figure 1B, Table S2). In order to further assess whether the presence of NETs in the tissue microenvironment was correlated with disease prognosis, the same sets of 80 tissue specimens were analyzed for correlation between the CitH3 level and the survival rate; the results indicated that patients with higher $\mathrm{CitH} 3$ tissue expression displayed a poorer survival percentage (Figure 1C). Collectively, these results showed that NETs formation was correlated with HCC malignant progression.

\section{Neutrophils of HCC Promote Cancer Cells Invasion via NETs Release in vitro}

In order to investigate the invasion-inducing capacity of neutrophils, we performed a transwell chamber assay.
Isolation of peripheral blood neutrophils from patients or healthy donors routinely yielded a purity of greater than $90 \%$, evaluated by white blood cell counting (Figure 2A, Table S1). Freshly isolated human neutrophils were plated into the lower chamber and cancer cells were added on the top of Matrigel-coated membranes in the upper wells. By co-culturing in this manner with HCC cells, neutrophils isolated from the HCC patients with metastasis effectively promoted HCC cell invasion, while those from an HD conversely inhibited the invasion phenotype (Figure 2B). Compared to HD neutrophils, HCC neutrophils exhibited an increased capacity of releasing the NETs structure into the extracellular space (Figure 2B). Here, we further observed that neutrophils from HCC patients with extrahepatic metastasis showed an enhanced capacity of forming NETs compared to those without metastasis (Figure 2D). These findings demonstrated that neutrophils from patients with HCC but not HD efficiently promoted HCC cell invasion.

In order to determine whether NETs promote HCC cell invasion, we digested extracellular NETs structure by adding DNase I into the transwell system. The ability of neutrophils to stimulate the invasion of $\mathrm{HuH} 7$ was inhibited by the digestion of DNase I, whereas DNase I displayed no effect on fetal bovine serum (FBS)-stimulated $\mathrm{HuH7}$ invasion. Next, the necessity of peptidyl arginine deiminase type 4 (PAD4), a crucial enzyme responsible for histone 
A

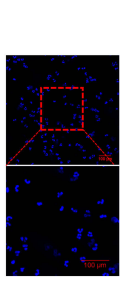

B

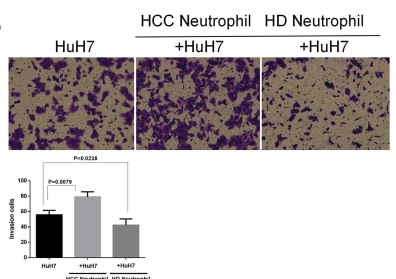

c

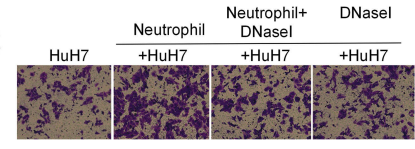

Pre treated Neutrophi

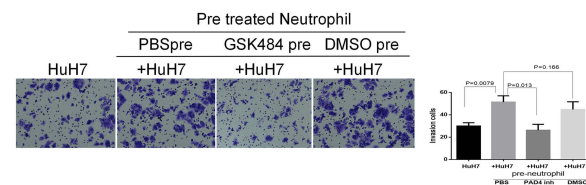

HuH7+Neutrophils from
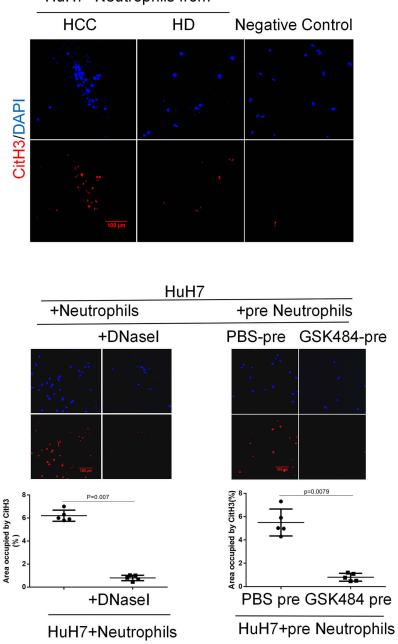

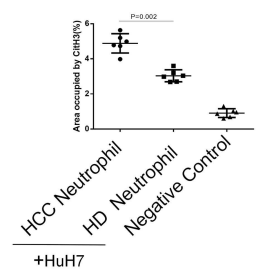

D

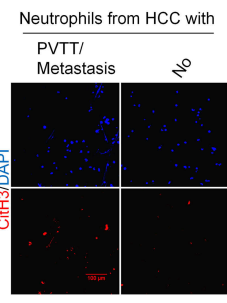

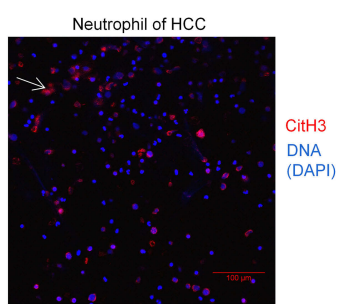

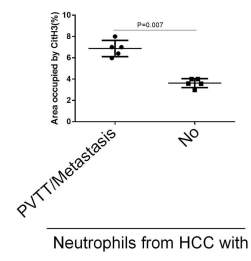

Figure 2 Neutrophil of HCC facilitates HCC cells invasion through NETs formation in vitro. (A) DAPI staining showed the purity of the neutrophils isolated from human peripheral whole blood. Scare bar: 100um. (B) The representative images of transwell assay for the effects of neutrophils on HCC cells invasion were shown. The cells were counted under a microscope in twenty randomly selected fields. NETs were identified by $\mathrm{CitH} 3$ component. The expression of NETs-CitH3 was detected by immunofluorescence staining on coverslip. The white arrow points to the expression and presence of NETs-CitH3. Scare bar: I00um. (C) The representative images of transwell assay for the effects of DNasel or PAD4 inhibitor GSK484 on neutrophils-induced HCC cells invasion were shown. The expression of NETs-CitH3 was detected by immunofluorescence staining on coverslip. Neutrophils of $\mathrm{HCC}$ was pre-treated with GSK 484 at I0uM for $4 \mathrm{~h}$ before co-culture. I5 $\mu \mathrm{L}$ DNasel was added into per $500 \mu \mathrm{L}$ culture medium in lower chamber. Scare bar: I00um. (D) The expression of NETs-CitH3 was detected by immunofluorescence staining for neutrophils from HCC patients

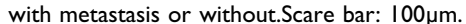

citrullination during NETosis, in NETs-stimulated cancer cell invasion was examined. As expected, pre-incubating neutrophils with the PAD4 inhibitor GSK484 before co-culture efficiently reduced the NETs-arousing capacity, which in turn blocked the neutrophils' ability to promote HCC cell invasion (Figure 2C). These results revealed that neutrophils of HCC, through NETs formation, contributed to HCC cell invasion.

\section{NETs Form in HCC Pre-Metastatic Niche, While the Neutrophils of HCC Enhance Metastasis in vivo}

HCC cells were intravenously injected into nude mice to establish a lung metastasis model. The success rate of this metastatic model reached about $83 \%$. In detail, sixteen out of twenty mice developed lung detectable metastasis foci after the injection of HCC cells. Using this mice model, injection of $\mathrm{HuH7}$ cells spontaneously resulted in gradual neutrophil infiltration into the site of the model lung at the pre-metastatic stage, in the absence of an infection trigger. We observed that neutrophil was the major immune component that accumulated and released the NETs structures in the lung, which preceded the microscopic metastasis foci. In detail, abundant neutrophil influx and NETs-CitH3 became detectable at about 40 days after injection, with the amounts elevating for days, whereas multiple cancer cells reached the inside of the lung and accumulated there about eight weeks later (Figure 3A and B; Figure S2). Accordingly, we speculated that neutrophils contributed to the formation of the premetastatic niche during HCC metastasis through the NETosis process. Next, to investigate the effect of HCC neutrophils on metastasis in vivo, we injected $\mathrm{HuH7}$ cells with or without human neutrophils of HCC intravenously into null mice. Enhanced metastatic burden was observed when $\mathrm{HuH} 7$ cells were co-injected with neutrophils, compared to the injection of $\mathrm{HuH} 7$ cells alone (Figure 3C). In addition, by tail vein injection of HCC cells with different metastatic capacity, we showed that the highly metastatic $\mathrm{HCC}$ cell line resulted in more ly- $6 \mathrm{G}^{+}$cells penetrating into the lung at the pre-metastasis stage (Figure 3D).

\section{HCC Cells-Derived CXCL8/IL-8 Primes Normal Neutrophils Towards NETs Formation in an NADPH-Dependent Manner}

We next focused on how the HCC cells elicited neutrophil sensitization for the formation of NETs. Indeed, cancer cell-induced NETs formation was dependent on the NADPH oxidase activity. ${ }^{10,42,49}$ We performed Western blot and immunohistochemistry assays, confirming that 
A
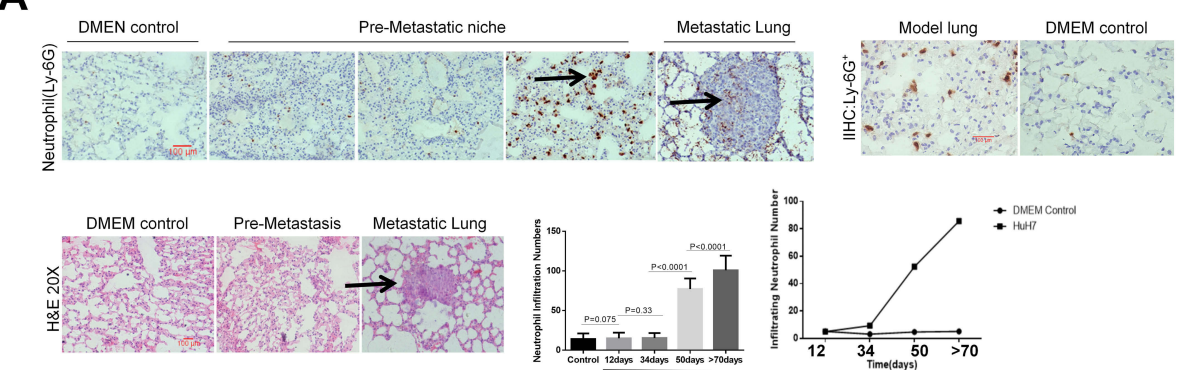

C

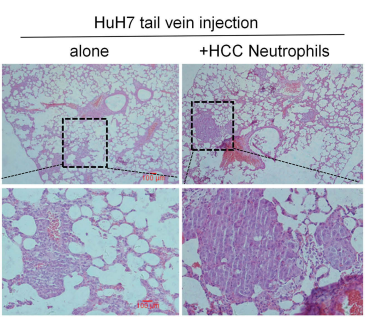

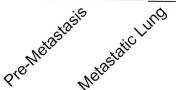

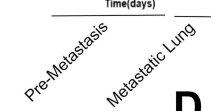

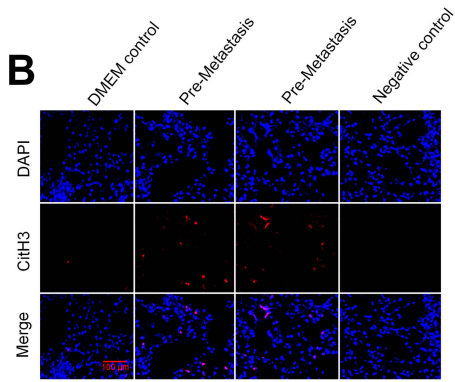
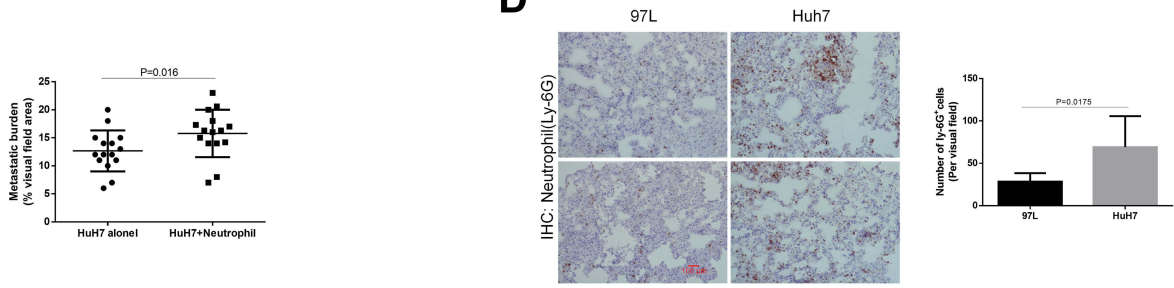

Figure 3 Neutrophils of HCC enhances metastasis burden in vivo. (A) HuH7 cells were injected into nude mice via tail vein. At various time points (I2, 34, 50days) after $\mathrm{HCC}$ cells injection, the mice was killed and examined the density of ly- $6 \mathrm{G}^{+}$cells infiltration. $\mathrm{Ly}-6 \mathrm{G}^{+}$cells in nude mice lung were shown by immunohistochemistry staining with lung frozen section ( $n=7$ each). Representative images of ly- $6 \mathrm{G}^{+}$neutrophils in the lung were shown. The black arrows point to $\mathrm{Ly}-6 \mathrm{G}^{+}$neutrophils (IHC staining) and metastasis (H\&E staining). Scare bar: $100 \mu \mathrm{m}$. (B) NETs-CitH3 were detected by immunofluorescence staining with lung frozen section. Representative images of CitH3 in the lung were shown $(n=7$ each). Scare bar: $100 \mu \mathrm{m}$. (C) HuH7 cells were co-injected with HCC neutrophils (I0:I) into nude mice ( $\mathrm{n}=7$ each). The metastatic burden in each group were calculated as the percentage of area occupied by metastatic tumor on each lung section, determined using Image J software. Representative images of metastatic foci in lung were shown. Scare bar: $100 \mu \mathrm{m}$. (D) $\mathrm{Ly}-6 \mathrm{G}^{+}$cells were detected by immunohistochemistry with frozen lung section after HuH7 or $97 \mathrm{~L}$ injection ( $\mathrm{n}=5$ each). Representative images were shown. Scare bar: $100 \mu \mathrm{m}$.

the pro-inflammatory cytokine IL-8 was produced remarkably in HCC cells and the patient's tissue, respectively (Figure 4A and B). By co-culturing HD neutrophils with the conditioned medium (CM) of HCC cells (HCC-CM), we observed that HCC-CM-induced HD neutrophils extruded extensive NETs, while the HD neutrophils alone exhibited a weak capacity of spontaneous NETs formation. Blockade of IL-8 with a neutralizing antibody $(\mathrm{Ab})$ led to a significant reduction in NETs formation, although not a complete inhibition, suggesting additional factors in HCC-CM being involved in NETs induction (Figure 4C).

Moreover, the activation of the NADPH oxidase (NOX2) enzyme in neutrophils has been shown to be essential for NETosis. ${ }^{15,16}$ A NOX2 inhibitor apocynin was previously found to strongly inhibit NETs formation and neutrophils-stimulated cancer cell invasion in vitro. ${ }^{46}$ As expected, inhibiting NOX2 with apocynin completely prevented the HCC-CM from inducing HD neutrophils toward NETs formation (Figure 4C). Likewise, robust NETs formation was aroused when HD neutrophils were co-cultured with HCC patient serum, but not normal serum. Consistently, IL-8-neutralizing Ab reduced seruminduced NETs formation, while NOX2 inhibitor apocynin exhibited a stronger inhibition capacity. But, treatment with apocynin did not completely prevent the NETs formation compared to the HD serum group (Fig. S1). Altogether, our data demonstrated that HCC cell-secreted CXCL8/IL-8 primed the HD neutrophils to release NETs structure through the NADPH pathway.

\section{NETs-Associated Cathepsin G Facilitates HCC Cells Invasion in vitro}

NETs are characterized by the association of neutrophil protease with extracellular DNA-binding histone. It is speculated that the pro-invasive effects of NETs require the NETs-associated protease activity. ${ }^{44,46}$ In order to elucidate what acts as a stimulus for the enhanced invasion phenotype of NETs-trapped HCC cells, we focused on the cathepsin $\mathrm{G}(\mathrm{cG})$ and neutrophil elastase (NE) components present on the NETs structure, providing a mechanistic insight by adding the indicated inhibitors into the coculture medium. Indeed, inhibition of $\mathrm{cG}$, but not $\mathrm{NE}$, efficiently blocked the neutrophils' ability to promote HCC cell invasion, and also strikingly reduced the extension of NETs formation (Figure 5B). These findings suggested that cG is not just an invasion driver; it is also 

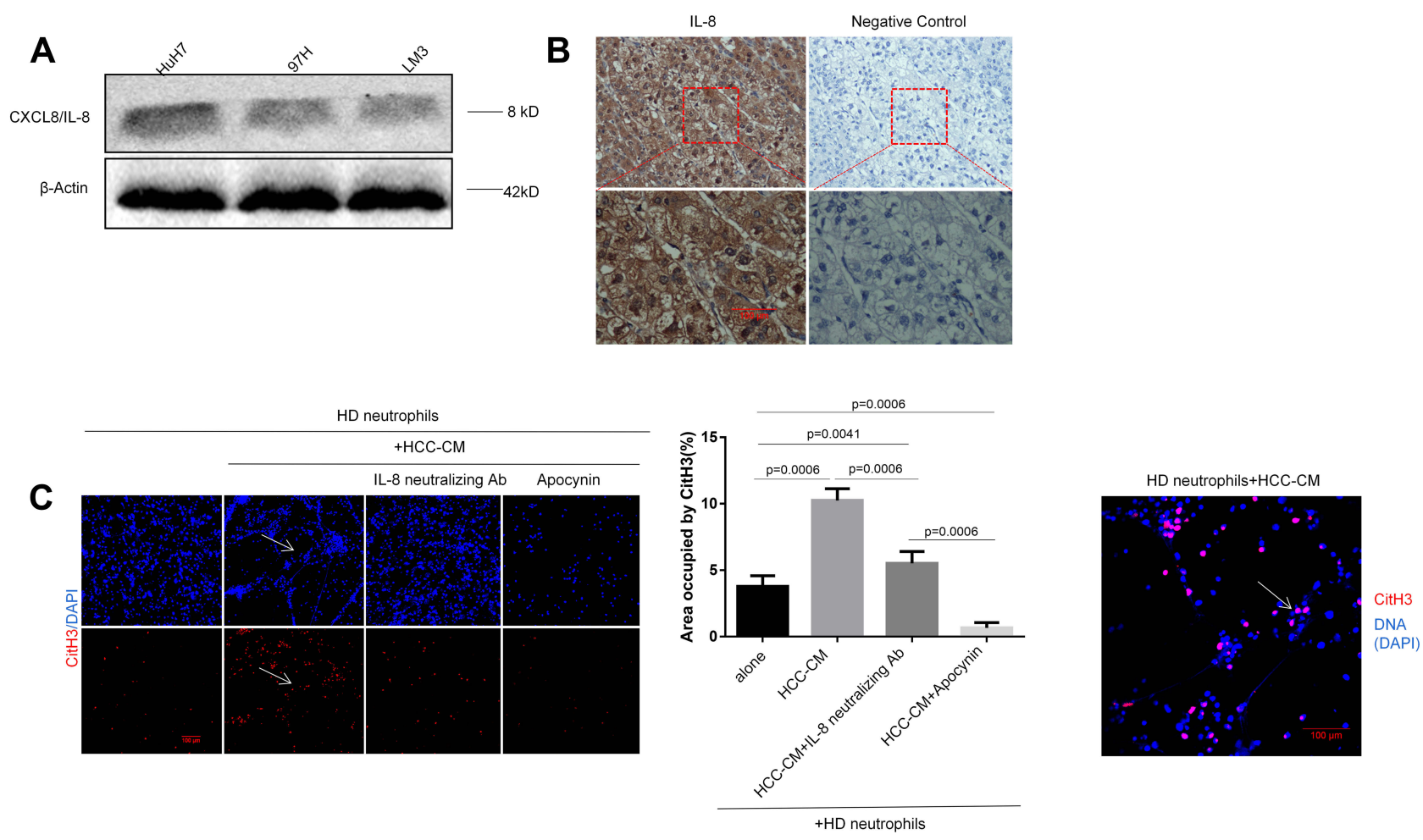

Figure 4 HCC cells-derived CXCL8/IL-8 triggers TAN towards NETs formation through NADPH pathway. (A) The protein expression of IL-8 was detected by Western blot assay in three HCC cell lines. Experiment was repeated three times. (B) The expression of IL-8 in HCC tissues was examined by the immunohistochemistry ( $\mathrm{n}=30$ ). A representative image was shown. Scare bar: 100 um. (C) Co-incubation of HD neutrophils $\left(2.5 \times 10^{6} / \mathrm{mL}\right)$ with the conditioned medium of $\mathrm{HCC}$ cells $(\mathrm{HCC}-\mathrm{CM})(\mathrm{I}: 2)$ was performed to explore the effect of IL-8 on HCC-CM-induced NETs formation. The expression of CitH3 was detected by immunofluorescence staining on coverslip for the identification of NETs formation. Representative images were shown. IL-8 neutralizing antibody was added into the culture medium at the concentration of $5 \mu \mathrm{g} / \mathrm{mL}$. NOX inhibitor apocynin was added into medium at the final concentration of IOuM. Experiment was repeated three times. Representative images were shown. The white arrows point to the presence of NETs-CitH3. Scare bar: $100 \mu \mathrm{m}$.

essential in the NETs extrusion. Conversely, NE inhibitor sivelestat showed no significant effect on the neutrophilsstimulated invasion phenotype.

Furthermore, we generated NETs-containing conditioned medium (NETs-CM) from HCC neutrophils that formed NETs in response to PMA (Figure 5A). Similarly, NETs$\mathrm{CM}$ markedly enhanced the invasion potential of HCC cells. Co-culturing NETs-CM with $\mathrm{cG}$ inhibitor equally blocked the invasion-arousing capacity (Figure 5C). As can be seen, $\mathrm{cG}$ protein was expressed during the PMA-stimulated NETosis process in vitro (Figure 6A); yet, digesting NETsDNA with DNaseI prevented invasion, although $\mathrm{cG}$ was not deleted by DNaseI digestion (Figure 6A-C). These results suggested that co-localization of the $\mathrm{cG}$ component with NETs-CitH3 was essential for inducing HCC cell invasion.

\section{Targeting the cG Component Effectively Reduces HCC Metastasis in vivo}

In order to test the effect of $\mathrm{cG}$ inhibitor on tumor metastasis in vivo, we established an experimental metastasis model by intravascular injection of Hep1-6 cells into C57BL/6J mice. In this model, NETs-CitH3 started to become detectable at the pre-metastatic stage in the model lung compared to the control arms, which could be abrogated by DNaseI treatment. However, as can be seen, the cG inhibitor exhibited little influence on the NETs formation in vivo, while significantly reducing the NETs-CitH3 release in vitro (Figure 7A). Thus, in line with the in vitro results, our findings indicated that the administration of either the $\mathrm{cG}$ inhibitor or the DNaseI treatment effectively led to significantly decreased lung metastasis burden compared to control arms (Figure 7B).

Clinically, with human HCC specimens, a higher density of $\mathrm{cG}$ protein was observed for metastatic patients than those without metastasis in the peritumoral tissues, but not in paracancerous tissues (Figure 8A and B). A Pearson correlation analysis confirmed that NETs-CitH3 expression positively correlated with $\mathrm{cG}$ in peritumoral tissues (Figure 8D). Moreover, by immunohistochemical staining, we found stronger co-localization between $\mathrm{cG}$ and $\mathrm{CitH} 3$ proteins in 
A

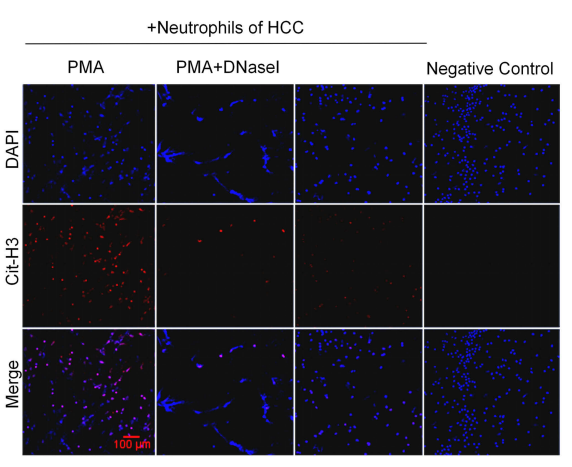

C

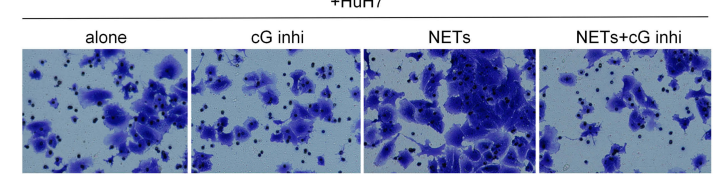

B
B $\mathrm{HuH7}$
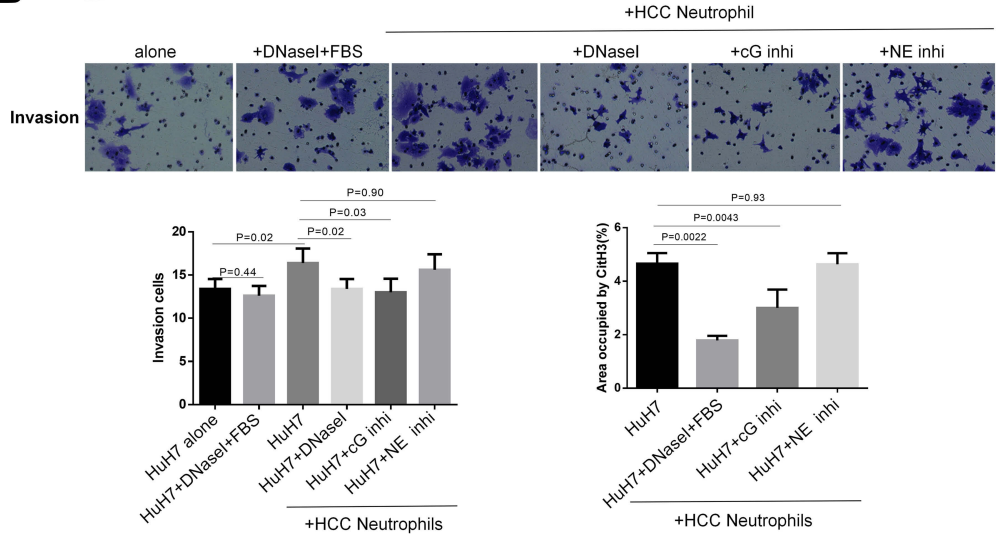

p=0.0071

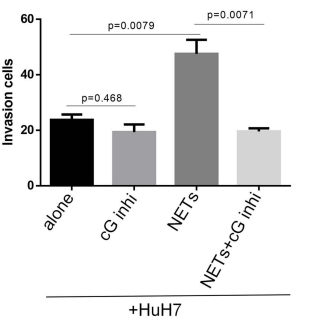

Figure 5 NETs-associated cathepsin G promotes HCC cells invasion. (A) Neutrophils generated NETs in response to PMA. PMA was co-incubated with neutrophils at a final concentration of $500 \mathrm{nM}$ for the preparation of NETs. DNasel abolished the process of PMA-induced NETs formation. The expression of CitH3 was detected by immunofluorescence staining on coverslip for the identification of NETs formation. Representative images were shown. Scare bar: I00 $\mu$ m. (B) cG inhibitor reduced NETs extension and neutrophils-induced HCC cells invasion. NE inhibitor had no significant effect on NETs formation and neutrophils-induced invasion. Representative images were shown. cG inhibitor was added into culture medium at a final concentration of $2 \mu \mathrm{M}$, and NE inhibitor was added into culture medium at a final concentration of IOuM. Experiment was repeated three times. (C) cG inhibitor abolished NETs-stimulated HCC cells invasion. cG inhibitor was added into culture medium at the final concentration of $2 \mu \mathrm{M}$. Control group was set with DMEM culture medium. Representative images were shown. Experiment was repeated three times.

the peritumoral tissue from metastatic HCC compared to the metastasis-free ones (Figure 8C). It is noteworthy that the higher density of co-localization indicated poor disease prognosis in HCC (Figure 8E). Collectively, these findings suggested the significant contribution of $\mathrm{cG}$ to $\mathrm{HCC}$ metastasis and that the cG inhibitor effectively prevented $\mathrm{HCC}$ lung metastasis in vivo.

\section{NETs-Associated Cathepsin G Regulates E-Cadherin Expression in HCC Cells}

It is reported that NETs-associated NE and MMP9 awaken dormant breast cancer cells by remodeling the extracellular matrix (ECM) ${ }^{5,19}$ In this study, we next focused on the mechanism by which NETs-associated cG facilitated HCC cell invasion. We hypothesized that a likely target for proteases is the adhesion molecule expressed on the surface of HCC cells. ${ }^{31,41,43}$ In order to test this hypothesis, $\mathrm{HCC}$ cell layers were incubated with NETs-CM for up to $20 \mathrm{~h}$; then, the depleted area of HCC cells was quantified. We observed that incubation with NETs-CM resulted in significant dyshesion of the HCC cell layers (Figure 9C). Using Western blotting, we analyzed the level of CXCL8/
IL-8 and E-cadherin in HCC cells under NETs stimulation, which revealed that NETs-containing CM stimulation for an indicated interval led to elevated expression of CXCL8/ IL-8 and decreased E-cadherin expression in HCC cells (Figure 9A and B). Immunofluorescence staining further confirmed the consistent alteration of E-cadherin protein on the surface of HCC cells (Figure 9D). Furthermore, the inhibitor of $\mathrm{cG}$ efficiently reversed the decline of E-cadherin but not of CXCL8/IL-8 (Figure 9A and B). Taken together, these results indicated that NETsassociated $\mathrm{cG}$ component promoted $\mathrm{HCC}$ cell invasion through decreasing E-cadherin expression in vitro.

\section{Discussion}

Tumor metastasis affects a significant proportion of patients with HCC. ${ }^{11,34}$ Here, our observations unveiled an inherent mechanism underlying the link between NETs and HCC progression. We began our investigations with the evaluation of human serum samples for the NETs marker MPO-DNA. We selected PVTT as an independent and critical indicator for HCC progression and found that patients with PVTT HCC developed higher NETs markers 

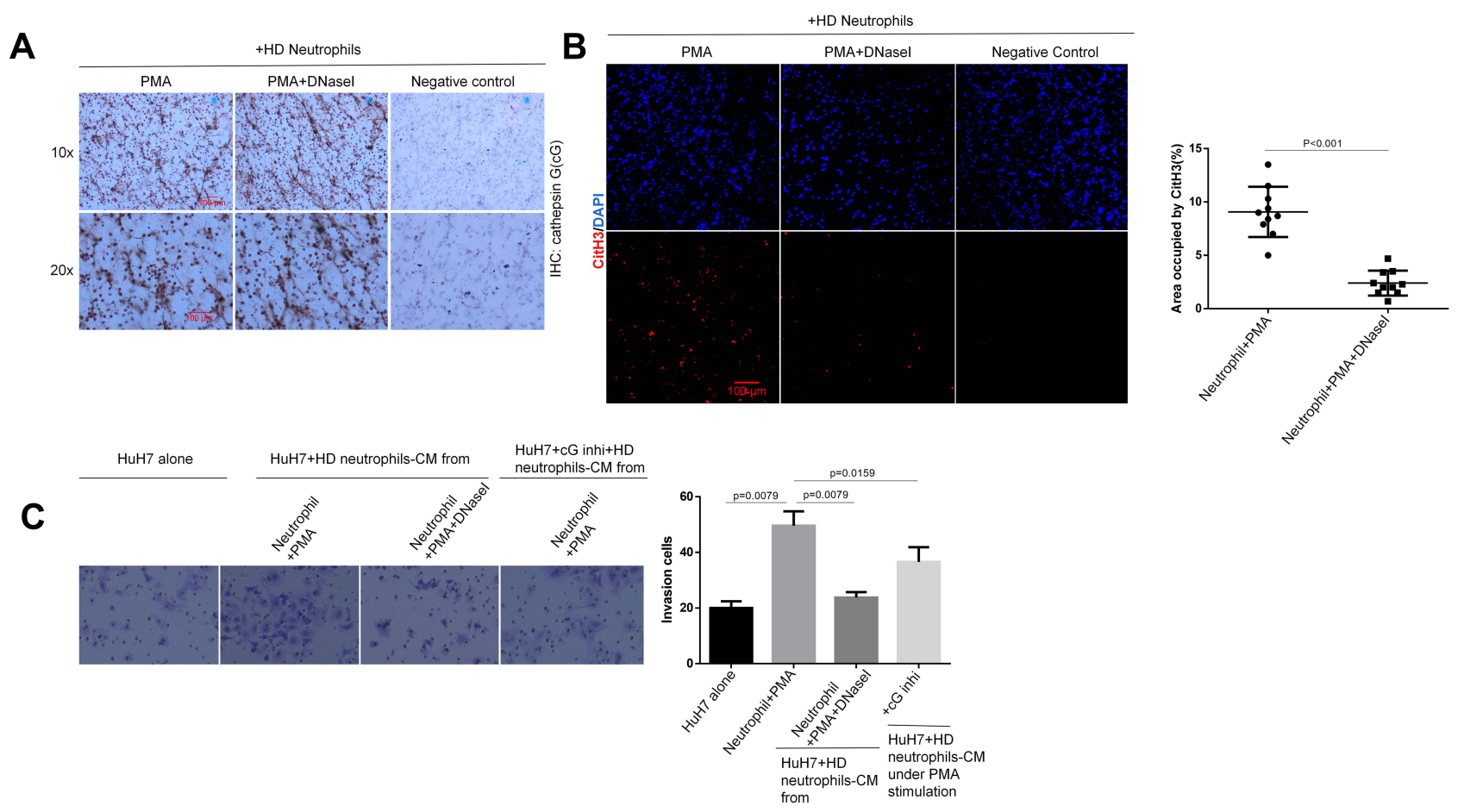

Figure 6 Co-localization of $\mathrm{cG}$ and $\mathrm{CitH} 3$ is necessary to promote invasion in vitro. (A) The cathepsin G expression of HD neutrophils on coverslip were shown by

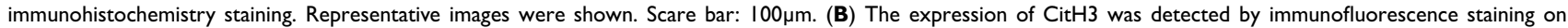

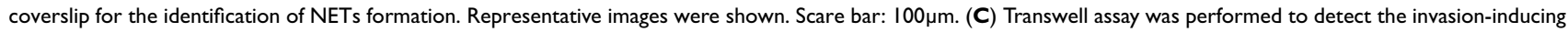
capacity of HD neutrophils-CM under different treatment. $c G$ inhibitor was added into culture medium at the final concentration of $2 \mu M$. Control group was set with DMEM culture medium. Representative images were shown. Experiment was repeated three times.

compared to those without PVTT. We obtained consistent results through all tumor-tissue specimens. These analyses supported our hypothesis of increased NETs formation in patients with advanced HCC.

It was initially suggested that NETs aided in the defense against bacteria, fungi, viruses, and parasites. ${ }^{2,3}$ However, if dysregulated, excessive NETs could contribute to several diseases, such as immune-related diseases and cancers. ${ }^{29,30,32}$ The relationship between NETsassociated proteins and cancer metastasis is interesting. Understanding more mechanisms can lead to the discovery of effective therapeutic targets in HCC progression. ${ }^{1,3,4}$ In this study, by blocking PAD4 and inhibiting $\mathrm{cG}$ in the transwell assay, we observed that neutrophils failed to enhance cancer cell invasion with reduced NETs formation, suggesting that PAD4 and $\mathrm{cG}$ mediated the key signaling pathways related to NETs-triggered invasion potential and HCC cells-inducing NETs release. We further demonstrated that NETs-derived $\mathrm{cG}$ promoted HCC cell invasion by reducing the levels of adhesion molecules and increasing the levels of inflammatory mediators in vitro. In addition to $\mathrm{cG}$, other peptides were released during NETosis. MMP9, one of the NETs- associated proteins, was initially demonstrated to promote the outgrowth of colorectal carcinoma micrometastasis after liver ischemia/reperfusion (I/R) injury. ${ }^{47}$ However, the mechanism by which these proteins/peptides participate in HCC metastasis in vivo remains to be investigated. $^{33,36}$

Inflammation has previously been reported to enhance cancer metastasis and is largely initiated by neutrophils. ${ }^{25,26}$ NETs formed in response to systemic bacterial infection or stress can enhance cancer cell dissemination in the metastatic microenvironment. ${ }^{20,21}$ Recently, a study has reported that increased NETs structure induced under inflammation conditions can promote HCC metastasis via pro-inflammatory TLR9-COX2 signaling pathways. In this study, lipopolysaccharide (LPS) injection into immune-component C57BL/6 mice was used to induce efficient NETs formation, which subsequently promoted lung metastasis. This study stressed the roles of inflammation factors in neutrophil-stimulated cancer invasion, and therefore, proposed that a combination of DNase I with the anti-inflammatory drug aspirin could serve as a novel therapeutic strategy against HCC metastasis. ${ }^{12}$ Although additional studies on the mechanisms of NETs 


\begin{tabular}{l}
0 day 2 day \\
\hline$\uparrow$
\end{tabular}

A
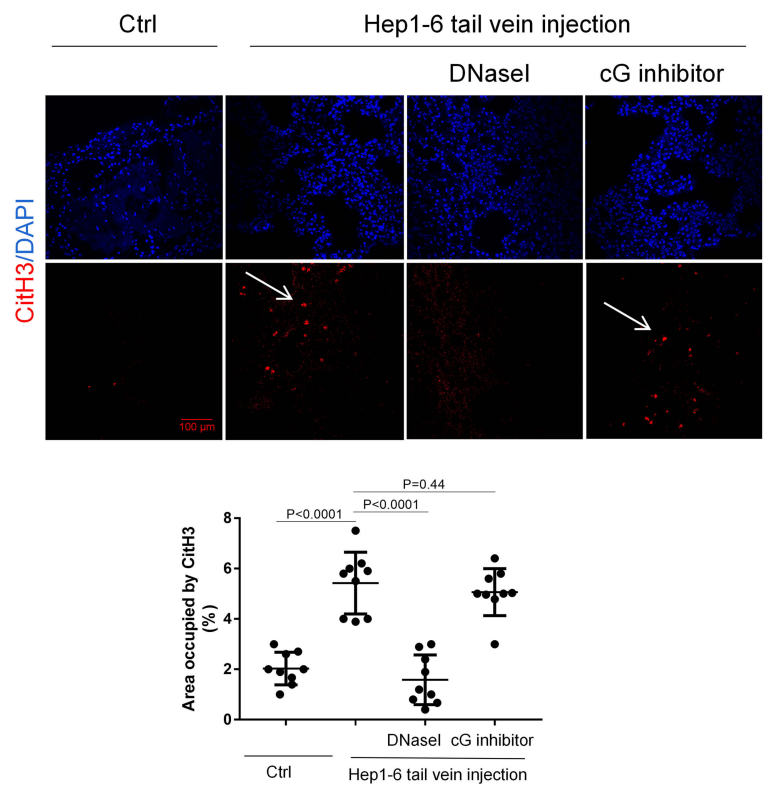

B

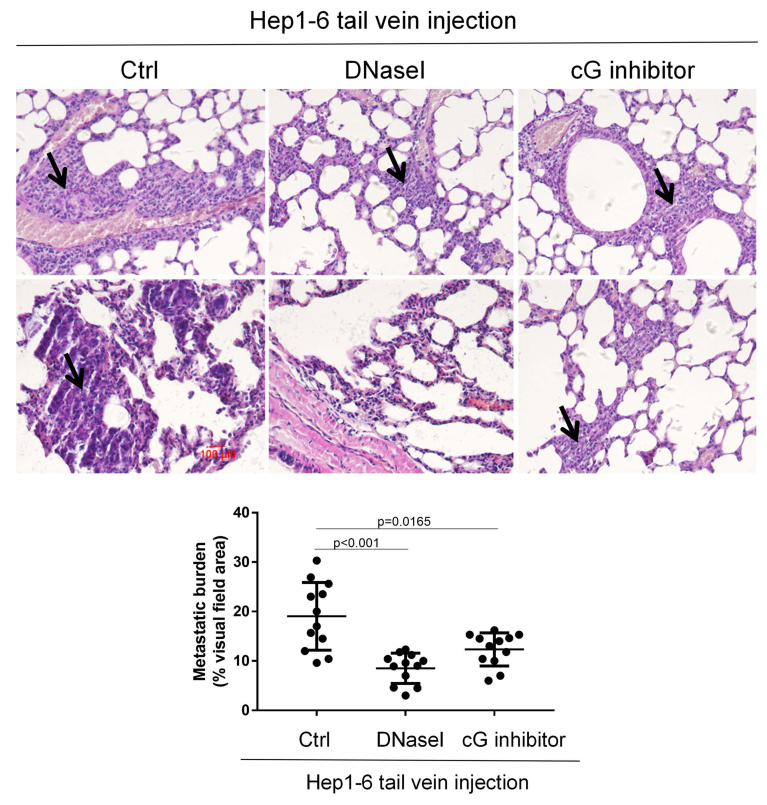

Figure 7 Inhibition of $\mathrm{cG}$ component alleviates lung metastasis in vivo. (A) The expression of NETs-CitH3 protein in model lung were shown by immunofluorescence ( $\mathrm{n}=5$

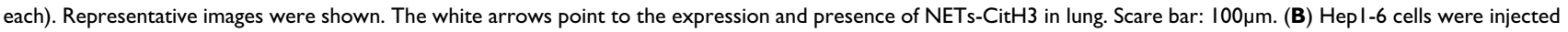
into male C57BL/6] mice via tail vein ( $n=5$ each), and the metastatic burden in each group were calculated as the percentage of area occupied by metastatic tumor on each

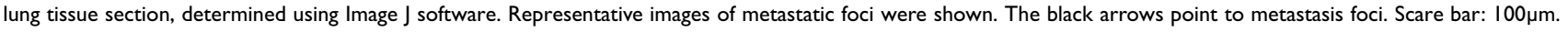

A

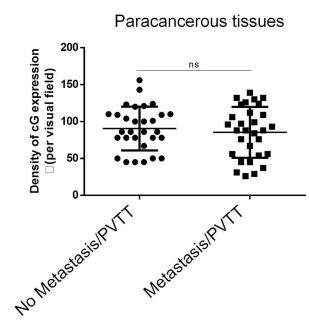

B

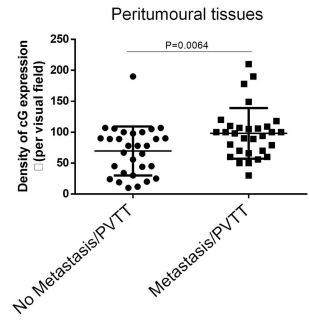

C

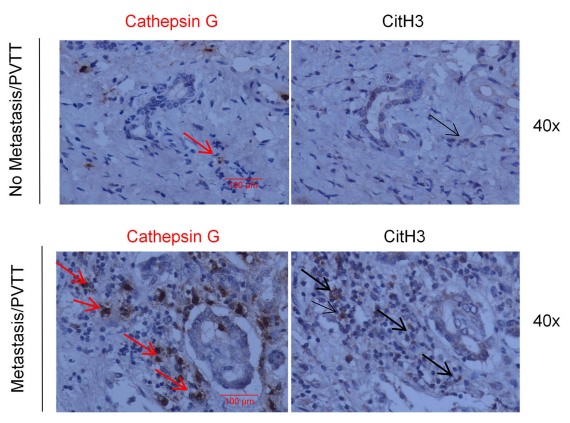

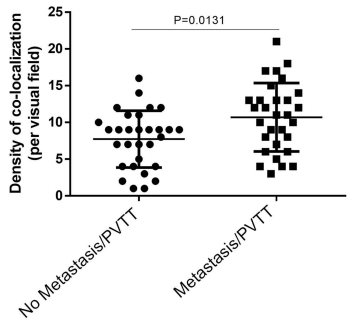

D

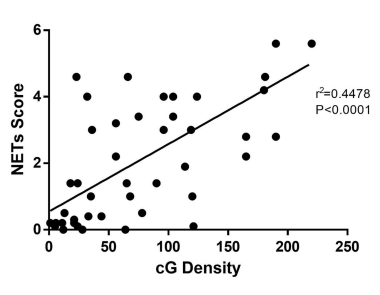

E

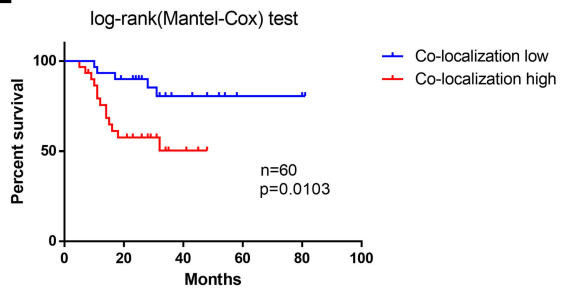

Figure 8 Co-localization between $\mathrm{CG}$ and NETs-CitH3 in HCC tissues indicates disease prognosis. (A-C) The expression of cathepsin G and CitH3 protein in patients tissues were shown by immunohistochemistry staining with serial sections $(n=60)$. Representative images were shown. The red arrows point to protein cathepsin $G$ and black arrows point to protein $\mathrm{CitH} 3$ in serial section, indicating the co-localization of protein cathepsin $\mathrm{G}$ and $\mathrm{CitH} 3$ in tissue. Scare bar: I00 $\mu$ m. (D) Pearson correlation analysis of NETs-CitH3 and cG expression in HCC samples $(n=60)$. (E) 60 non-metastasis/PVTT HCC patients with different level of cG and NETs for metastasis/PVTT cumulative incidence analysis, and Log rank test was used. 


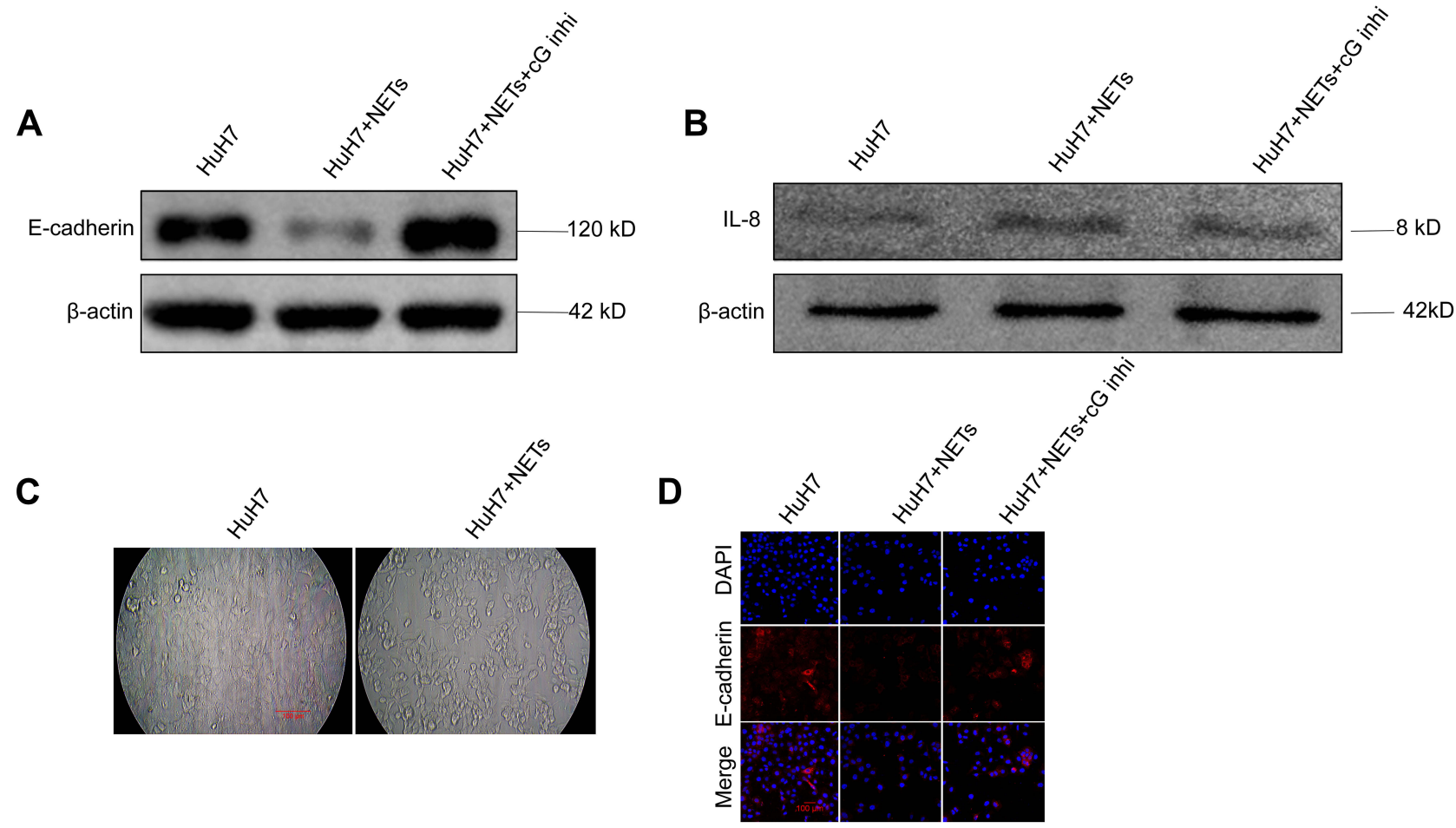

Figure 9 NETs-associated cathepsin G regulates the expression of E-cadherin in HCC cells in vitro. (A, B) Western blot analysis of E-cadherin and CXCL8/IL-8 level in $\mathrm{HuH7}$ cells treated with NETs-CM. Cells were stimulated with NETs-CM for 20 hours. Experiment was repeated three times. (C) HuH7 (5xI0 $0^{5}$ cells) were co-cultivated with NETs-CM or cultivated alone to confluence for 20 hours, and the area depleted of tumor cells was quantified as percentage of the total area. Representative images were shown. Scare bar: $100 \mu m$. (D) E-cadherin expression of HuH7 cells layer was shown by immunofluorescence staining. Representative images were shown. Scare bar: I00um. Experiment was repeated three times.

in cancer progression are needed, some significant progress, particularly in the metastasis and invasion fields, had been made. There has been another strong evidence that NETs triggered HMGB1 release and activated TLR9-dependent signaling pathway in cancer cells to enhance liver metastasis potential after surgical stress in colorectal cancer. $^{47}$ Therefore, whether the HMGB1-TLR9-COX2 signaling axis is affected in cancer metastasis deserves additional investigation.

In the present study, we demonstrated a previously unrecognized pathway on how NETs induced HCC metastasis as well as how HCC cells triggered NETs formation. We found that HCC neutrophils enhanced HCC cell invasion through NETs release in vitro. In line with this, the human HCC neutrophils elicited the HCC cell metastasis in vivo. Then, we sought to determine, which factors secreted from cancer cells accounted for increased NETs formation. Of note, the NETs-arousing ability of HCC-CM was observed to be reduced upon IL-8 blockade. We have shown that HCC-secreted IL-8 triggered neutrophils to initiate the NETosis process through the NADPH oxidase pathway, featuring a new mechanism between HCC cells and their microenvironment. The dissemination of $\mathrm{HCC}$ cells has a strong predilection for the lung and has often been described as a passive process that is, in part, governed by the microenvironmental mechanisms. ${ }^{37-39}$ Using the nude mice metastatic model, we observed that HCC cell injection could spontaneously result in neutrophil influx and NETs formation in the pre-metastatic lung in the absence of inflammation induction. Our findings suggested that NETs formation and neutrophil influx induced by cancer cells may be a critical driver for subsequent metastasis behavior, which could be prevented by DNase I. Altogether, with the release of all the pro-tumorigenic peptides, NETs act as fertilizers enriching the premetastatic niche to facilitate cancer metastasis in HCC.

NETs, with their large, extracellular, web-like structures, are composed of several granule proteins that are assembled on a scaffold of extruded DNA. These proteins are mainly histones, neutrophil elastase, cathepsin G, myeloperoxidase, defensins, and actin. ${ }^{3}$ However, how the NETs composition impacts the NETs function in diseases remains to be identified. We, therefore, determined the $\mathrm{cG}$ protein on the PMA-stimulated NETs structure and then 
explored the roles of $\mathrm{cG}$ in NETs-induced HCC cell invasion in vitro. Our results indicated that co-localization expression of $\mathrm{cG}$ and $\mathrm{CitH} 3$ was necessary for promoting HCC cell invasion. Using the HCC tissue samples, the colocalization expression between $\mathrm{cG}$ and NETs-CitH3 was also observed in the peritumoral tissues, and the density of co-localization was observed to be correlated with disease prognosis. These findings suggested $\mathrm{cG}$ as a key signaling target in NETs-triggered metastasis in vivo.

\section{Conclusions}

In summary, our research identified a tumor-associated cellular, molecular, and clinical network, involving NETs, cG, NADPH signaling, and IL-8, that regulates the positive feedback loop between neutrophils and cancer progression. These findings supported the idea of the exploration of this network as a potential therapeutic target against HCC progression.

\section{Abbreviations}

NETs, neutrophils extracellular traps; IL-8, interleukin-8; HCC, hepatocellular carcinoma; CM, conditioned medium; cG, Cathepsin G; PVTT, Portal vein tumor thrombosis; NE, neutrophil elastase; PAD4, peptidyl arginine deiminase type 4 .

\section{Data Sharing Statement}

All data generated or analyzed during this study are included in this published article.

\section{Ethics Approval}

Samples were collected after approval by the medical ethics committee of Zhongshan Hospital affiliated to Xiamen University and written informed consents were obtained from each patient. All animal experiments were approved by the animal Ethics committee of Xiamen University, and all institutional and national guidelines for the care and use of laboratory animals were followed.

\section{Consent for Publication}

All authors have seen and approved the manuscript and consent for publication.

\section{Acknowledgments}

This work was supported by grants from the National Natural Science Foundation of China (NO. 81871961, 81871963 and 81802344) and Medical and Health Key project of Xiamen (3502Z20191106).

\section{Author Contributions}

Xiangqian Guan performed the study, acquisition and analysis of data, and drafted the manuscript. Yuyan Lu participated in animal study and protein assays. Heping Zhu carried out the collection of patients blood specimens. Wenxiu Zhao participated in draft writing and data analysis. Xiaoqin Chi provided tissues specimens and clinicopathological characteristics of HCC patients. Shuqi Yu participated in the preparation of tissue sections, and helped to perform functional experiments and immunoassays. Chengrong Xie participated in data analysis and gave some technique assistance. Zhenyu Yin started the study and participated in experimental design. Chengrong Xie and Zhenyu Yin approved for the final version and submission. All authors made a significant contribution to the work reported, whether that is in the conception, study design, execution, acquisition of data, analysis and interpretation, or in all these areas; took part in drafting, revising or critically reviewing the article; gave final approval of the version to be published; have agreed on the journal to which the article has been submitted; and agree to be accountable for all aspects of the work.

\section{Disclosure}

The authors reported no conflicts of interest for this work.

\section{References}

1. Guo L, Chen G, Zhang W, et al. A high-risk luminal A dominant breast cancer subtype with increased mobility. Breast Cancer Res Treat. 2019;175(2):459-472.

2. Berger-Achituv S, Brinkmann V, Abed UA, et al. A proposed role for neutrophil extracellular traps in cancer immunoediting. Front Immunol. 2013;4:48.

3. Papayannopoulos V. Neutrophil extracellular traps in immunity and disease. Nat Rev Immunol. 2018;18(2):134-147.

4. Quail DF, Joyce JA. Microenvironmental regulation of tumor progression and metastasis. Nat Med. 2013;19(11):1423-1437.

5. Albrengues J, Shields MA, Ng D, et al. Neutrophil extracellular traps produced during inflammation awaken dormant cancer cells in mice. Science. 2018;361:6409.

6. Llovet JM, Montal R, Sia D, Finn RS. Molecular therapies and precision medicine for hepatocellular carcinoma. Nat Rev Clin Oncol. 2018;15(10):599-616.

7. Van der Windt DJ, Sud V, Zhang H, et al. Neutrophil extracellular traps promote inflammation and development of hepatocellular carcinoma in nonalcoholic steatohepatitis. Hepatology. 2018;68(4):1347-1360.

8. Gomes T, Várady CBS, Lourenço AL, et al. IL-1 $\beta$ blockade attenuates thrombosis in a neutrophil extracellular trap-dependent breast cancer model. Front Immunol. 2019;10:2088.

9. Lee W, Ko SY, Mohamed MS, Kenny HA, Lengyel E, Naora H. Neutrophils facilitate ovarian cancer premetastatic niche formation in the omentum. $J$ Exp Med. 2019;216(1):176-194.

10. An Z, Li J, Yu J, et al. Neutrophil extracellular traps induced by IL-8 aggravate atherosclerosis via activation NF- $\mathrm{kB}$ signaling in macrophages. Cell Cycle. 2019;18(21):2928-2938. 
11. Zhou SL, Yin D, Hu ZQ, et al. A positive feedback loop between cancer stem-like cells and tumor-associated neutrophils controls hepatocellular carcinoma progression. Hepatology. 2019;70(4):1214-1230.

12. Yang LY, Luo Q, Lu L, et al. Increased neutrophil extracellular traps promote metastasis potential of hepatocellular carcinoma via provoking tumorous inflammatory response. J Hematol Oncol. 2020;13(1):3.

13. Zhou SL, Zhou ZJ, Hu ZQ, et al. Tumor-associated neutrophils recruit macrophages and T-regulatory cells to promote progression of hepatocellular carcinoma and resistance to sorafenib. Gastroenterology. 2016;150(7):1646-1658.

14. Leal AC, Mizurini DM, Gomes T, et al. Tumor-Derived exosomes induce the formation of neutrophil extracellular traps: implications for the establishment of cancer-associated thrombosis. Sci Rep. 2017;7(1):6438.

15. Yuzhalin AE. Citrullination in Cancer. Cancer Res. 2019;79 (7):1274-1284.

16. Wen F, Shen A, Choi A, Gerner EW, Extracellular SJ. DNA in pancreatic cancer promotes cell invasion and metastasis. Cancer Res. 2013;73(14):4256-4266.

17. Mascaux C, Angelova M, Vasaturo A, et al. Immune evasion before tumour invasion in early lung squamous carcinogenesis. Nature. 2019;571(7766):570-575.

18. Manley HR, Keightley MC, Lieschke GJ. The neutrophil nucleus: an important influence on neutrophil migration and function. Front Immunol. 2018;9:2867.

19. Cedervall J, Zhang Y, Olsson AK. Tumor-Induced NETosis as a risk factor for metastasis and organ failure. Cancer Res. 2016;76 (15):4311-4315.

20. El Rayes T, Catena R, Lee S, et al. Lung inflammation promotes metastasis through neutrophil protease-mediated degradation of Tsp-1. Proc Natl Acad Sci U S A. 2015;112(52):16000-16005.

21. Kim J, Bae JS. Tumor-associated macrophages and neutrophils in tumor microenvironment. Mediators Inflamm. 2016;2016:6058147.

22. Seys SF, Lokwani R, Simpson JL, Bullens DMA. New insights in neutrophilic asthma. Curr Opin Pulm Med. 2019;25(1):113-120.

23. Demers M, Wagner DD. NETosis: a new factor in tumor progression and cancer-associated thrombosis. Semin Thromb Hemost. 2014;40 (3):277-283.

24. Morimoto-Kamata R, Yui S. Insulin-like growth factor-1 signaling is responsible for cathepsin G-induced aggregation of breast cancer MCF-7 cells. Cancer Sci. 2017;108(8):1574-1583.

25. McLoed AG, Sherrill TP, Cheng DS, et al. Neutrophil-derived IL-1 $\beta$ Impairs the efficacy of NF- $\mathrm{B}$ inhibitors against lung cancer. Cell Rep. 2016;16(1):120-132.

26. Yang Z, Guo J, Weng L, Tang W, Jin S, Ma W. Myeloid-derived suppressor cells-new and exciting players in lung cancer. $J$ Hematol Oncol. 2020;13(1):10.

27. Jimenez RV, Kuznetsova V, Connelly AN, Hel Z, Szalai AJ. C-reactive protein promotes the expansion of myeloid derived cells with suppressor functions. Front Immunol. 2019;10:2183.

28. Kapanadze T, Gamrekelashvili J, Ma C, et al. Regulation of accumulation and function of myeloid derived suppressor cells in different murine models of hepatocellular carcinoma. J Hepatol. 2013;59(5):1007-1013.

29. Liu Y, Liu L. The pro-tumor effect and the anti-tumor effect of neutrophils extracellular traps. Biosci Trends. 2020;13(6):469-475.

30. He YM, Li X, Perego M, et al. Transitory presence of myeloid-derived suppressor cells in neonates is critical for control of inflammation. Nat Med. 2018;24(2):224-231.

31. Gan WJ, Wang JR, Zhu XL, et al. RAR $\gamma$-induced E-cadherin downregulation promotes hepatocellular carcinoma invasion and metastasis. J Exp Clin Cancer Res. 2016;35(1):164.

32. Pieterse E, Rother N, Garsen M, et al. Neutrophil extracellular traps drive endothelial-to-mesenchymal transition. Arterioscler Thromb Vasc Biol. 2017;37(7):1371-1379.
33. Lerman I, Hammes SR. Neutrophil elastase in the tumor microenvironment. Steroids. 2018;133:96-101.

34. Ng CKY, Di Costanzo GG, Terracciano LM, Piscuoglio S. Circulating cell-free DNA in Hepatocellular carcinoma: current insights and outlook. Front Med (Lausanne). 2018;5:78.

35. Banini BA, Sanyal AJ. The use of cell free DNA in the diagnosis of HCC. Hepatoma Res. 2019;5:34.

36. Schön MP, Erpenbeck L. The interleukin-23/interleukin-17 axis links adaptive and innate immunity in psoriasis. Front Immunol. 2018;9:1323.

37. Angelova M, Mlecnik B, Vasaturo A, et al. Evolution of metastases in space and time under immune selection. Cell. 2018;175 (3):751-765.

38. Mishalian I, Bayuh R, Eruslanov E, et al. Neutrophils recruit regulatory T-cells into tumors via secretion of CCL17-a new mechanism of impaired antitumor immunity. Int J Cancer. 2014;135(5):1178-1186.

39. He W, Xu J, Mu R, et al. High-salt diet inhibits tumour growth in mice via regulating myeloid-derived suppressor cell differentiation. Nat Commun. 2020;11(1):1732.

40. Demers M, Krause DS, Schatzberg D, et al. Cancers predispose neutrophils to release extracellular DNA traps that contribute to cancer-associated thrombosis. Proc Natl Acad Sci U S A. 2012;109 (32):13076-13081. doi:10.1073/pnas.1200419109

41. Martins-Cardoso K, Almeida VH, Bagri KM, et al. Neutrophil Extracellular Traps (NETs) Promote pro-metastatic phenotype in human breast cancer cells through epithelial-mesenchymal transition. Cancers (Basel). 2020;12(6):1542.

42. Teijeira Á, Garasa S, Gato M, et al. CXCR1 and CXCR2 chemokine receptor agonists produced by tumors induce neutrophil extracellular traps that interfere with immune cytotoxicity. Immunity. 2020;52 (5):856-871.

43. Padmanaban V, Krol I, Suhail Y, et al. E-cadherin is required for metastasis in multiple models of breast cancer. Nature. 2019;573 (7774):439-444.

44. Yang L, Liu Q, Zhang X, et al. DNA of neutrophil extracellular traps promotes cancer metastasis via CCDC25. Nature. 2020;583 (7814):133-138.

45. Zheng C, Zheng L, Yoo JK, et al. Landscape of infiltrating T cells in liver cancer revealed by single-cell sequencing. Cell. 2017;169 (7):1342-1356.

46. Park J, Wysocki RW, Amoozgar Z, et al. Cancer cells induce metastasis-supporting neutrophil extracellular DNA traps. Sci Transl Med. 2016;8(361):361ra138.

47. Tohme S, Yazdani HO, Al-Khafaji AB, et al. Neutrophil Extracellular Traps Promote the Development and Progression of Liver Metastases after Surgical Stress. Cancer Res. 2016;76(6):1367-1380.

48. Honda M, Kubes P. Neutrophils and neutrophil extracellular traps in the liver and gastrointestinal system. Nat Rev Gastroenterol Hepatol. 2018;15(4):206-221.

49. Alfaro C, Teijeira A, Oñate C, et al. Tumor-produced Interleukin-8 attracts human myeloid-derived suppressor cells and elicits extrusion of Neutrophil Extracellular Traps (NETs). Clin Cancer Res. 2016;22 (15):3924-3936.

50. Mauracher LM, Posch F, Martinod K, et al. Citrullinated histone H3, a biomarker of neutrophil extracellular trap formation, predicts the risk of venous thromboembolism in cancer patients. $J$ Thromb Haemost. 2018;16(3):508-518.

51. Deng Q, Pan B, Alam HB, et al. Citrullinated Histone H3 as a therapeutic target for endotoxic shock in mice. Front Immunol. 2020;9(10):2957. 


\section{Publish your work in this journal}

The Journal of Hepatocellular Carcinoma is an international, peerreviewed, open access journal that offers a platform for the dissemination and study of clinical, translational and basic research findings in this rapidly developing field. Development in areas including, bu not limited to, epidemiology, vaccination, hepatitis therapy, pathology and molecular tumor classification and prognostication are all considered for publication. The manuscript management system is completely online and includes a very quick and fair peer-review system, which is all easy to use. Visit http://www.dovepress.com/ testimonials.php to read real quotes from published authors.

Submit your manuscript here: https://www.dovepress.com/journal-of-hepatocellular-carcinoma-journal 